\title{
Endorsement of Compulsory HIV Vaccination Policy among Populations at High Risk of HIV Exposure (LA VOICES)
}

\author{
Peter A. Newman ${ }^{1,{ }^{*}}$, Sung-Jae Lee ${ }^{2}$, Ellen T. Rudy ${ }^{3}$, Allison Diamant ${ }^{4}$, Naihua Duan ${ }^{5}$, Terry \\ Nakazano $^{6}$, and William E. Cunningham ${ }^{7}$ \\ ${ }^{1}$ Factor-Inwentash Faculty of Social Work, University of Toronto, Ontario, Canada \\ ${ }^{2}$ Department of Psychiatry and Biobehavioral Sciences, UCLA Semel Institute, Center for \\ Community Health, University of California, Los Angeles, California, USA \\ ${ }^{3}$ Los Angeles County Department of Health Services, Sexually Transmitted Disease Program, \\ Los Angeles, California, USA \\ ${ }^{4}$ Ronald Reagan UCLA Medical Center, University of California, Los Angeles, California, USA \\ ${ }^{5}$ Department of Psychiatry, Columbia University Medical Center, New York, USA \\ ${ }^{6}$ Division of General Internal Medicine \& Health Services Research, UCLA David Geffen School \\ of Medicine, University of California, Los Angeles, California, USA \\ ${ }^{7}$ Department of Health Policy and Management, UCLA Fielding School of Public Health, \\ University of California, Los Angeles, California, USA
}

\begin{abstract}
Compulsory vaccination is a frequently implemented policy option for ensuring comprehensive vaccine coverage. Ongoing controversies around human papillomavirus vaccine dissemination, and suboptimal coverage, suggest the value of assessing acceptability of compulsory vaccinations — particularly among likely target populations—in advance of their public availability to support evidence-informed interventions. With the first HIV vaccine to demonstrate partial efficacy in a large-scale clinical trial, we examined individual characteristics and attitudes associated with support for compulsory HIV vaccination policy among a diverse, representative sample of adults attending probable HIV vaccine dissemination venues in a large urban county. Participants were recruited using three-stage probability sampling from likely venues for future HIV vaccine dissemination. We used Audio-CASI to administer a 60-minute structured questionnaire. Items included endorsement of compulsory HIV vaccination policy, sociodemographic characteristics, injecting drug use, vaccine attitudes and perceived HIV risk. Among 1225 participants (mean age $=36.8$ years; $55.6 \%$ males, $37.6 \%$ non-English speaking Hispanic, $78.8 \%$ heterosexual, $25.7 \%$ injection drug users), almost half (48.2\%) endorsed a compulsory HIV vaccination policy. NonEnglish speaking Hispanics compared to whites, participants with less than high school education, higher positive vaccine attitude scores and higher perceived HIV risk were significantly more likely, and people who inject drugs significantly less likely to endorse compulsory HIV
\end{abstract}

*Corresponding author: Peter A. Newman, Factor-Inwentash Faculty of Social Work, University of Toronto, 246 Bloor Street West, Toronto, Ontario, Canada M5S 1V4; Tel: +1 416-946-8611; Fax: +1 416-978-7072; p.newman@utoronto.ca. 
vaccination. Public health interventions to promote positive vaccine attitudes and accurate perceptions of HIV risk among vulnerable populations, and strategies tailored for people who inject drugs, may build support for compulsory HIV vaccination policy and promote broad HIV vaccine coverage.

\section{Keywords}

HIV vaccines; vaccination; policy; vulnerable populations; attitudes

Vaccinations against infectious diseases have been lauded as one of the greatest public health achievements in the past century in dramatically improving health and life expectancy in the United States (US) (Centers for Disease Control and Prevention [CDC], 1999). As we enter the $21^{\text {st }}$ century, much of the morbidity and health costs incurred in the US are attributable to HIV and other sexually transmitted infections (STI) (Chesson et al., 2011), for which we have few vaccinations (Workowski \& Berman, 2010). Compulsory vaccination is a frequently implemented policy option for ensuring high population levels of vaccination. However, there has been little empirical study of the acceptability of compulsory vaccinations for preventable infectious diseases, particularly STIs. In the context of ongoing controversies around human papillomavirus (HPV) vaccine dissemination (Gabriel \& Grady, 2011; Haber, Marlow, \& Zimet, 2007) and anti-vaccine conspiracy theories (Bearman, 2010; Roberts, Newman, Duan, \& Rudy, 2005), assessing public perceptions and beliefs regarding the acceptability of compulsory vaccinations-in advance of their public availability - may support evidence informed dissemination strategies. Public reactions and social and policy challenges, as well as the public health impact, are likely to be particularly potent in the case of vaccines against HIV infection.

Compulsory HIV vaccination has the potential to yield the most comprehensive vaccine coverage. However, the implementation of compulsory HIV vaccination without prior assessment of public opinion-particularly among those for whom vaccinations are mandated - may lead to community backlash that seriously compromises the dissemination process. In the case of HPV vaccination, several U.S. state legislatures and governing bodies attempted to pass compulsory middle school vaccination policies; these have ignited fervent public and political opposition from various constituencies (Gabriel \& Grady, 2011; National Conference of State Legislatures [NCSL], 2013). In response, a number of states altered their policies. For example, Texas overturned the governor's executive order for compulsory vaccination; and Virginia approved generous "opt-out" provisions (NCSL, 2013). While the potential benefits of HPV vaccines for preventing cervical cancer are not questioned and, undoubtedly, compulsory HPV vaccination would boost vaccination coverage, the pace of dissemination has been slowed. A dissemination plan based on systematic research in regard to the public's beliefs and concerns about compulsory HPV vaccination may have averted some of the polarizing debates that ensued, thus facilitating more widespread acceptance and, in turn, more comprehensive vaccine coverage.

HIV, similar to HPV, disproportionately affects marginalized populations in the US, such as African Americans and Latinos (CDC, 2012a). Understanding perceptions of possible 
compulsory HIV vaccine policies among these ethnic minority communities, as well as other populations at high risk for HIV exposure such as people who inject drugs, may support successful HIV vaccine policy and dissemination.

Several investigations have assessed factors that influence acceptance of mandatory schoolbased vaccinations among parents (Ferris, Horn, \& Waller, 2010; Kennedy, Brown, \& Gust, 2005; Salmon et al., 2005). A nationally representative survey of U.S. parents $(n=1527)$ indicates that more positive beliefs about vaccines, belief that vaccines are necessary to prevent certain diseases and that vaccines are important to children's health are associated with support of compulsory school vaccination (Kennedy, Brown, \& Gust, 2005). Conversely, in a survey of parents $(\mathrm{n}=815)$ in five U.S. states who claimed non-medical exemptions for their school-age children for a broad range of mandatory vaccinations, the most common reasons were low perceived susceptibility and low perceived vaccine efficacy and safety (Salmon et al., 2005).

In the case of adults, the Advisory Committee on Immunization Practices (ACIP) and a variety of healthcare organizations support mandatory seasonal influenza vaccination for healthcare workers $(\mathrm{HCW})$, particularly given three decades of low compliance with voluntary vaccination (Derber \& Shankaran, 2012; Ottenberg et al., 2011). Although mandatory vaccination policies have been shown to be the strongest predictor of compliance with seasonal influenza vaccine uptake among both hospital-based and nonhospital-based HCW's (n=3188) (Rebmann, Wright, Anthony, Knaup, \& Peters, 2012), institutional and $\mathrm{HCW}$ resistance to such policies persist, with few published studies of HCW's perspectives on such mandates (Derber \& Shankaran, 2012; Ottenberg et al., 2011).

Assessment of attitudes toward compulsory HIV vaccination among populations at elevated risk of HIV exposure, and in settings that might serve as venues for vaccine delivery, may support effective interventions to facilitate future HIV vaccine dissemination. The objective of this study is to examine individual characteristics and attitudes associated with support for compulsory HIV vaccination among an ethnically, socially and economically diverse, representative sample of adults attending probable HIV vaccine dissemination venues in a large urban county.

\section{Methods}

\section{Participants}

Participants at risk for HIV in Los Angeles (LA) County ( $n=1225)$ were recruited using three-stage probability sampling. In stage I, we randomly selected sites from three venuebased strata: 1) LA County sexually transmitted disease (STD) clinics ( $\mathrm{n}=12)$; 2) Latino community-based organizations (CBOs) offering HIV testing and other health-related services $(n=8)$; and 3 ) needle exchange programs (NEP) providing clean needles or syringes in exchange for dirty needles $(n=8)$. Probability-proportional-to-estimated size sampling assigned the sampling probability for each site to be proportional to its estimated client load. Stage II consisted of randomly selecting sessions of approximately four hours in duration (morning, afternoon, or evening) within each site. A total of 75 sessions from each stratum 
were sampled ( 225 sessions across 3 strata). In stage III, we randomly selected participants within each session at selected sites.

Eligibility criteria were being at least 18 years of age, not an employee of the recruitment site, and not known to be HIV-positive. Following informed consent, participants were reimbursed $\$ 20$ for engaging in a one-time, 60 -minute interview administered by trained interviewers using laptop computers programmed with Questionnaire Development System (QDS) software (NOVA Research Company). Sensitive questions on sexual risk behaviors and drug use were administered by Audio Computer-assisted Self-interviewing (ACASI), an approach successfully used in our formative research (Newman et al., 2006a; Newman et al., 2007). The study protocol was reviewed and approved by the Human Subjects Protection Committees of UCLA and the Los Angeles County Department of Public Health, and the Research Ethics Board of the University of Toronto. All participants provided written informed consent.

\section{Measures}

Main outcome-Compulsory HIV vaccine policy endorsement was the main outcome of interest. A dichotomous HIV vaccine policy endorsement variable was constructed from three items assessing whether respondents agreed about: "everyone to be vaccinated," "all school children to be vaccinated," and "all adults to be vaccinated" (for HIV). These items were asked on a 5-point Likert-type response scale from "strongly agree" to "strongly disagree"; "strongly agree" or "somewhat agree" was considered to be agreement. Participants who agreed to at least one of the three statements were considered to endorse compulsory HIV vaccine policy. Participants who disagreed in response to all three statements were considered to oppose compulsory HIV vaccine policy.

Sample characteristics-Sociodemographic characteristics collected for participants during the 60-minute interview were age, gender, race/ethnicity, sexual orientation, education, having children, monthly income, health insurance and access to medical care. The description of how the sample characteristics are measured is provided in Table 1.

Independent variables-The positive vaccine attitude scale was the average score of 7 vaccine attitude items reflecting positive attitudes towards vaccines, including an HIV vaccine (Cronbach's $a=0.61$ ). The items in the positive vaccine attitude scale were 4-point Likert responses to the statements: "I think it is a good idea to get flu shots," "Existing vaccines have worked well to protect people against many diseases such as small pox and tetanus," "Vaccines are important to protect children," "Vaccines are important to keep me healthy," "Everyone who gets an AIDS vaccine will be protected against HIV/AIDS," "If I get an AIDS vaccine, I can lower chances of getting infected with HIV/AIDS," and " I would be one of the first people to get an AIDS vaccine."

Perceived HIV risk was assessed with the statement "I am worried about getting HIV/ AIDS." Responses were measured on a 5-point Likert scale.

Injecting drug use was assessed by asking participants whether they injected drugs in the past 30 days (yes/no). 


\section{Data Analysis}

In order to account for the three-stage probability sampling strategy used in the study, all analyses were conducted using STATA 9.0 (College Station, TX) and its survey commands to adjust for the sampling strata, primary sampling units (PSU) and population weights. This approach permits the findings from the sample to represent the source population of persons at risk attending sites within the three strata.

Prior to estimating statistical models, distributional assumptions of the models were assessed. With only 2 missing observations (on the positive vaccine attitudes scale), we did not address non-random missingness explicitly. In order to avoid severe collinearity problems, we used a variance inflation factor (VIF) with a cutoff value of less than 4.0; as standard error is doubled with VIF $=4.0$, this is a commonly used criterion to determine if any factors are highly correlated with the other factors (Fox, 1991). None of the variables were highly correlated. Exploratory bivariate analyses computing the Pearson chi-square statistic were performed to examine the associations of sociodemographic characteristics, vaccine attitudes and compulsory HIV vaccine policy endorsement. We then estimated a multivariable logistic regression model to examine the association between the outcome of compulsory HIV vaccine policy endorsement and the sociodemographic and independent variables. Variables were included in the model based on findings from the bivariate analyses ( $\mathrm{P}$-value < 0.1), in addition to variables selected a priori based on previous research (i.e., "age" and "have a regular place to go for medical care"). A systematic review of 20 studies recommended the inclusion of age as a demographic in future investigations of HIV vaccine acceptability (Newman \& Logie, 2010). The accessibility of medical care and vaccination, in addition to mandatory influenza vaccination policies, have been associated with seasonal influenza, including H1N1, vaccine compliance (Rebmann et al., 2012). Additionally, we tested possible interactions between race/ethnicity and vaccine attitudes, and IDU by perceived HIV risk, on endorsement of compulsory HIV vaccine policy. Weighted odds ratios (OR) and 95\% confidence intervals (CI) were computed for each variable in the multivariable logistic regression model.

\section{Results}

The mean age of participants $(\mathrm{N}=1225)$ was 36.8 years. Male participants made up $55.6 \%$ of the sample; Hispanics (non-English speaking) were 37.6\%; and heterosexuals were 78.8\%. Almost one-third (29.5\%) reported having less than high school education; $54.1 \%$ had children; and almost half (49.2\%) had a monthly income of $\$ 1000$ or less. Over two-thirds $(68.6 \%)$ reported having a regular place to go for medical care, but nearly half $(48.8 \%)$ were uninsured.

Table 1 highlights the bivariate associations of sociodemographic characteristics, vaccines attitudes, and perceived risk with compulsory HIV vaccine policy endorsement. About half $(\mathrm{n}=590,48.2 \%)$ endorsed a compulsory HIV vaccine policy. Females (54.0\%) were more likely than males $(43.3 \%)$ to endorse a compulsory HIV vaccine policy ( $\mathrm{p}=0.001)$. Race/ ethnicity was significantly associated with compulsory HIV vaccine policy endorsement $(\mathrm{p}<0.001)$. Other sociodemographic characteristics significantly associated with compulsory HIV vaccine policy endorsement were being heterosexual (50.8\%) compared with gay or 
bisexual (38.0\%) ( $\mathrm{p}=0.002)$; lower educational attainment $(\mathrm{p}<0.001)$; having children $(\mathrm{p}=0.001)$; and being uninsured $(\mathrm{p}=0.022)$. Positive vaccine attitudes $(\mathrm{p}<0.001)$ and perceived HIV risk $(\mathrm{p}<0.001)$ were significantly and positively associated with compulsory HIV vaccine policy endorsement. People who reported injecting drugs in the past 30 days were significantly less likely (34.1\%) than non-injection drug users $(52.9 \%)$ to endorse compulsory HIV vaccine policy $(\mathrm{p}<0.001)$.

Table 2 outlines the results of the multivariable logistic regression examining predictors of compulsory HIV vaccine policy endorsement. Consistent with the bivariate analyses, the following demographic characteristics were significantly associated with compulsory HIV vaccine policy endorsement: non-English speaking Latinos compared to whites ( $\mathrm{OR}=1.69$; 95\% CI: 1.03, 2.76; $\mathrm{p}=0.037$ ); having less than high school education, compared to bachelor's degree or above ( $\mathrm{OR}=2.66$; 95\% CI: 1.56, 4.55; $\mathrm{p}<0.001)$; and having some college education, compared to bachelor's degree or above (OR=1.71; 95\% CI: 1.03, 2.86; $\mathrm{p}=0.039)$. Participants who scored higher on the positive vaccine attitude scale were significantly more likely to endorse compulsory HIV vaccine policy $(\mathrm{OR}=2.48 ; 95 \% \mathrm{CI}$ : $1.70,3.61 ; \mathrm{p}<0.001)$. In addition, those with higher levels of perceived HIV risk were significantly more likely to endorse compulsory HIV vaccine policy $(\mathrm{OR}=1.17 ; 95 \% \mathrm{CI}$ : $1.04,1.32 ; \mathrm{p}=0.008)$. People who inject drugs were significantly less likely than noninjecting drug users to endorse a compulsory HIV vaccine policy ( $\mathrm{OR}=0.66$; 95\% CI: 0.45 , $0.97 ; \mathrm{p}=0.036)$. The race/ethnicity-by-positive vaccine attitudes interaction term $(\mathrm{OR}=1.21$; 95\% CI: 0.91, 1.59; $\mathrm{p}=0.18)$ and IDU-by-perceived HIV risk interaction term $(\mathrm{OR}=1.02$; $95 \%$ CI: $0.75,1.39 ; \mathrm{p}=0.89$ ) were not significant when considered in the final model.

\section{Discussion}

Compulsory HIV vaccination was endorsed by just under half of this sample of racially/ ethnically diverse adults from vulnerable communities at risk for HIV infection. Our sample was designed to represent likely priority populations for future HIV vaccine dissemination. Lack of support for a compulsory HIV vaccination policy among likely recipients could deter dissemination efforts. Evidence of low Hepatitis B virus (HBV) vaccination coverage among populations at high risk before the implementation of universal infant HBV vaccination policy in the U.S. indicates that efforts to vaccinate adults in a voluntary targeted manner may result in suboptimal uptake (CDC, 2006). Sufficient HIV vaccine coverage may need to rely on some sort of compulsory vaccine policy. However, governmental and public health implementation of a compulsory HIV vaccination policy without an understanding of target populations' attitudes that may limit their acceptance of the policy may jeopardize successful implementation, as witnessed with HPV vaccine dissemination efforts (NCSL, 2013; CDC, 2012b).

In this study we assessed compulsory HIV vaccine policy acceptance among different groups at elevated risk for contracting HIV and examined acceptance in terms of people's attitudes and behaviors. We found that persons with positive vaccine attitudes, persons with higher perceived risk for HIV, non-English speaking Latinos, heterosexuals and less educated persons were more likely to endorse a compulsory HIV vaccine policy. People who inject drugs were less likely to endorse a compulsory HIV vaccine policy. 
Increasing the public's attention to the positive impact vaccinations overall have had in improving health and well-being in the US and globally may lay the foundation for greater acceptance of a compulsory HIV vaccination policy. Our data supports the concept that people with favorable attitudes toward vaccines in general are more likely to endorse compulsory HIV vaccination. This is consistent with other studies in which positive attitudes about vaccination were associated with higher pneumococcal and influenza vaccination acceptance rates by adults classified as high-risk for either of these vaccine-preventable infections (Nichol, Mac Donald, \& Hauge, 1996). Public and patient education and the media may play an important role in facilitating favorable attitudes towards vaccines, in general, and specifically in the case of HIV vaccines.

Our findings indicate that persons with higher perceived risk of acquiring HIV are more likely to agree to a compulsory HIV vaccination policy, a finding consistent with the Health Belief Model (Rosenstock, Strecher, \& Becker, 1994). Higher perceived risk for HIV is associated with greater acceptance of HIV prevention technologies, such as HIV testing (CDC, 2002a; McGarrigle et al., 2005; Thomas et al., 2008), although HIV stigma is negatively associated with HIV testing uptake regardless of perceived risk (Fortenberry, McFarlane, \& Bleakley, 2002). Education and social marketing campaigns that promote accurate perceptions of risk among populations at high risk for HIV exposure may increase their willingness to support a compulsory HIV vaccination policy. Additionally, a broad based compulsory vaccination policy in the case of HIV may have corollary benefits in mitigating the stigma likely to be associated with HIV vaccination (Newman, Duan, Rudy, Roberts, \& Swendeman, 2004), which may further promote uptake.

In this study, people who inject drugs were strongly opposed to any type of compulsory HIV vaccination policy, which may create challenges in the acceptance and dissemination of HIV vaccines. People who inject drugs are at significantly elevated risk for HIV infection (CDC, 2012c) and are often homeless or near homeless, enmeshed in their addiction, publicly admonished, sometimes incarcerated and as such often have little trust in the government and other authorities (CDC, 2002b; Rhodes, Singer, Bourgois, Friedman, \& Strathdee, 2005). Future HIV vaccine dissemination policies may benefit from paying careful attention to attitudes toward vaccination and issues of trust (i.e., in government) of marginalized groups in order to facilitate receipt of vaccination rather than counterproductively engendering apprehension and distrust (Roberts et al., 2005).

Our findings did not demonstrate any difference in acceptance of compulsory HIV vaccination policy for African Americans and English-speaking Latinos compared to whites. Given the disproportionate prevalence of HIV in the African American community, these findings do not portend opposition to an HIV vaccine from African Americans; but further work is still necessary to increase endorsement among a community that is traditionally underserved by the healthcare system. Spanish-speaking Latinos in this study endorsed a compulsory HIV vaccination policy, which suggests some cross-cultural variation both within and across ethnicity. A statewide study of HPV vaccine acceptability, also conducted in California, suggests higher acceptability among Hispanics versus whites, however results were not disaggregated by primary language (Constantine \& Jerman, 2007). The lack of a significant ethnicity-by-vaccine attitude interaction on endorsement of compulsory HIV 
vaccination in the present study, in light of the narrow confidence intervals, suggests that rather than merely an inconclusive finding, we can rule out the possibility of large interaction effects. Thus other factors may influence differential endorsement by ethnicity. Future investigations of the impact of language and other cultural differences, as suggested for HPV vaccine promotion (Lechuga, Swain \& Weinhardt, 2011) and HIV vaccine trial preparedness (Newman et al., 2006b), may have important implications for the design of culturally tailored interventions to support HIV vaccine dissemination. This also serves as a reminder that dissemination efforts must be conducted in multiple languages.

The history of HBV vaccination in the US may provide relevant lessons for the present study. Soon after the vaccine against HBV was licensed in 1982, the ACIP recommended vaccination for adults at high risk for HBV infection. This risk-targeted approach missed a substantial proportion of the high risk, marginalized population and made no impact on the transmission of the disease; the incidence of hepatitis B infection remained constant (13.2 cases per 100,000) (CDC, 2002c). Over 30\% of these cases could not be attributed to any risk factor (Shapiro, 1993). Ultimately, universal childhood and compulsory middle school vaccination against $\mathrm{HBV}$ was implemented in the US, with powerful results (CDC, 2006). Surveillance data from a Florida middle school, for example, indicated an over four-fold increase in HBV vaccination coverage in the year after the state legislated compulsory middle school HBV vaccination compared with the year prior to the state's endorsement of the policy (CDC, 1998). Overall, as a result of these new strategies, HBV infection declined $75 \%$ in the US from 1990-2004, and infection in children comprised $94 \%$ of that decline (CDC, 2006). This history of HBV vaccines suggests that careful planning for HIV and other STI vaccine dissemination should include an evaluation of potential outcomes to vaccine policy implementation proposals as well as ongoing monitoring of policy outcomes and the willingness to change strategies to optimize vaccination coverage.

There are some limitations to this study to consider. For one, stated intentions are imperfect proxies for actual behavior; however, we aimed to assess attitudes toward compulsory HIV vaccination policy before rollout of a licensed vaccine. Second, the present sample, while representative of adults attending venues in Los Angeles that provide services to a range of persons at elevated risk for HIV infection, may not be generalizable to other locales or populations; further research on the acceptability of compulsory HIV vaccination policies among adults, and adolescents, in other HIV epicenters is warranted. Third, in any survey self-report data on sensitive items, such as injection drug use, may be underreported, which might impact on the results. However we used ACASI for sensitive items, which has been shown to improve the quality of assessments of sensitive risk behaviors (Metzger et al., 2000); and the present analysis had less than $1 \%$ missing data. Additionally, in the case of people who inject drugs, we identified a significant negative association with endorsement of compulsory HIV vaccination. These factors suggest that missing data were unlikely to have significantly affected the results. Fourth, the positive vaccine attitudes scale we used was not a standardized scale and was developed by our group based on factor analysis of vaccine attitude items; the Cronbach's alpha of 0.61 is modest at best. This underscores the lack of reliable standardized scales in the field to measure vaccine attitudes. Fifth, we observed a somewhat nonlinear relationship between endorsement of compulsory HIV vaccine policy and education: those with less than high school and some college, compared 
to those with a bachelor's degree, reported higher odds of endorsing compulsory HIV vaccine policy. However, those with high school education did not have higher odds of endorsement compared to those with a bachelor's degree. This could be due to potential misclassification between the "high school" and "some college" groups. In general, our findings support the notion that those with less education tended to have higher odds of endorsing compulsory HIV vaccine policy.

Lastly, our outcome was the sum of both adult and childhood compulsory HIV vaccination policies. This combination scale may skew the true acceptance of one policy option. However, we conducted sensitivity analysis and considered the outcome by each of the groups they were endorsing (all children, everyone, all adults). The findings from the multivariable logistic regression models were robust and consistent with the results from the single dichotomous outcome variable.

HIV vaccine dissemination will inevitably be a volatile public health issue. A compulsory HIV vaccination policy may be slow to emerge; but the lessons learned from the failures of targeted voluntary vaccination efforts with HBV vaccination and the heated debate over dissemination of HPV vaccines, along with slow roll-out and suboptimal coverage, implicate the need for early discussions and research about the acceptability of proposed policies. HIV vaccine policy decisions should be based on dynamic and emerging biological, behavioral and social evidence. In the wake of the first candidate HIV vaccine to demonstrate efficacy in a large-scale clinical trial (Rerks-Ngarm et al., 2009), this study provides preliminary information about the attitudes and sociodemographic characteristics associated with acceptance of a compulsory HIV vaccination policy among vulnerable populations at risk for HIV in the US. The time to develop the groundwork for HIV vaccine policy decisions is not when a vaccine is commercially ready; attitudes take time to change and delayed uptake would result in unnecessary morbidity and mortality. With no abatement of HIV incident infections in the US in the past decade and over 1 million people living with HIV (Hall, Song, \& Rhodes, 2008), developing evidence informed policy decisions and strategies in advance of product availability is crucial to ensuring efficient rollout of HIV vaccines.

\section{References}

Bearman P. Just-so stories: Vaccines, autism, and the single-bullet disorder. Social Science Quarterly. 2010; 73:112-115.

Centers for Disease Control and Prevention. Effectiveness of a seventh grade school entry vaccination requirement—statewide and Orange County, Florida, 1997-1998. Morbidity and Mortality Weekly Report. 1998; 47:711-715. Retrieved from http://www.cdc.gov/mmwr/preview/mmwrhtml/ 00054618.htm. [PubMed: 9746399]

Centers for Disease Control and Prevention. Ten great public health achievements-United States, 1900-1999. Morbidity and Mortality Weekly Report. 1999; 48:241-243. Retrieved from www.cdc.gov/mmwr/preview/mmwrhtml/00056796.htm. [PubMed: 10220250]

Centers for Disease Control and Prevention. Unrecognized HIV infection, risk behaviors, and perceptions of risk among young black man who have sex with men—six U.S. cities, 1994-1998. Morbidity and Mortality Weekly Report. 2002a; 51:733-736. Retrieved from http://www.cdc.gov/ mmwr/preview/mmwrhtml/mm5133a1.htm. [PubMed: 12201605]

Centers for Disease Control and Prevention. Substance abuse treatment and public health: working together to benefit injection drug users. 2002b. Retrieved from www.cdc.gov/idu/facts/ workingtogetherFin.pdf 
Centers for Disease Control and Prevention. Achievements in public health: Hepatitis B vaccinationUnited States, 1982-2002. Morbidity and Mortality Weekly Report. 2002c; 51:549-552,563.

Retrieved from www.cdc.gov/mmwr/preview/mmwrhtml/mm5125a3.htm. [PubMed: 12118536]

Centers for Disease Control and Prevention. Hepatitis B vaccination coverage among adults-United States, 2004. Morbidity and Mortality Weekly Report. 2006; 55:509-511. Retrieved from http:// www.cdc.gov/mmwr/preview/mmwrhtml/mm5518a3.htm. [PubMed: 16691181]

Centers for Disease Control and Prevention. Estimated HIV incidence among adults and adolescents in the United States, 2007-2010. HIV Surveillance Supplemental Report. 2012a; 17:14-22. Retrieved from http://www.cdc.gov/hiv/pdf/statistics_hssr_vol_17_no_4.pdf.

Centers for Disease Control and Prevention. Teen vaccination coverage: 2011 National Immunization Survey (NIS) - Teen. 2012b. Retrieved from www.cdc.gov/vaccines/who/teens/vaccinationcoverage.html

Centers for Disease Control and Prevention. HIV infection and HIV-associated behaviors among injecting drug users-20 cities, United States, 2009. Morbidity and Mortality Weekly Report. 2012c; 61:133-138. Retrieved from http://www.cdc.gov/mmwr/preview/mmwrhtml/ mm6108a1.htm. [PubMed: 22377843]

Chesson HW, Gift TL, Owusu-Edusei K Jr. Tao G, Johnson AP, Kent CK. A brief review of the estimated economic burden of sexually transmitted diseases in the United States: Inflationadjusted updates of previously published cost studies. Sexually Transmitted Diseases. 2011; 38:889-891. [PubMed: 21934557]

Constantine NA, Jerman P. Acceptance of human papillomavirus vaccination among Californian parents of daughters: A representative statewide analysis. Journal of Adolescent Health. 2007; 40:108-115. [PubMed: 17259050]

Derber CJ, Shankaran S. Health-care worker vaccination for influenza: Strategies and controversies. Current Infectious Disease Reports. 2012; 14:627-632. [PubMed: 22941054]

Ferris D, Horn L, Waller JL. Parental acceptance of a mandatory human papillomavirus (HPV) vaccination program. Journal of the American Board of Family Medicine. 2010; 23:220-229. [PubMed: 20207933]

Fortenberry JD, McFarlane M, Bleakley A. Relationships of stigma and shame to gonorrhea and HIV screening. American Journal of Public Health. 2002; 92:378-381. [PubMed: 11867314]

Fox, J. Regression Diagnostics. Sage; Newberry Park, CA: 1991.

Gabriel, T.; Grady, D. In Republican race, a heated battle over the HPV vaccine. 2011. Retrieved from www.nytimes.com/2011/09/14/us/politics/republican-candidates-battle-over-hpv-vaccine.html

Haber G, Marlow RM, Zimet GD. The HPV vaccine mandate controversy. Journal of Pediatric and Adolescent Gynecology. 2007; 20:325-331. [PubMed: 18082853]

Hall HI, Song R, Rhodes P. Estimation of HIV Incidence in the United States. Journal of the American Medical Association. 2008; 300:520-529. [PubMed: 18677024]

Kennedy AM, Brown CJ, Gust DA. Vaccine beliefs of parents who oppose compulsory vaccination. Public Health Reports. 2005; 120:252-258. [PubMed: 16134564]

Lechuga J, Swain GR, Weinhardt LS. The cross-cultural variation of predictors of human papillomavirus vaccination intention. Journal of Women's Health. 2011; 20:225-230.

McGarrigle CA, Mercer CH, Fenton KA, Copas AJ, Wellings K, Erens B, Johnson AM. Investigating the relationship between HIV testing and risk behavior in Britain: National survey of sexual attitudes and lifestyles 2000. AIDS. 2005; 19:77-84. [PubMed: 15627036]

Metzger DS, Koblin B, Turner C, Navaline H, Valenti F, Holte S, et al. Randomized controlled trial of audio computer-assisted self-interviewing: Utility and acceptability in longitudinal studies. American Journal of Epidemiology. 2000; 152:99-106. [PubMed: 10909945]

National Conference of State Legislatures. HPV vaccine. 2013. Retrieved from www.ncsl.org/issuesresearch/health/hpv-vaccine-state-legislation-and-statues.aspx

Newman PA, Duan N, Lee SJ, Rudy E, Seiden D, Kakinami L, Cunningham WE. HIV vaccine acceptability among communities at risk: The impact of vaccine characteristics. Vaccine. 2006a; 24:2094-2101. [PubMed: 16332402] 
Newman PA, Duan N, Lee SJ, Rudy ET, Seiden D, Kakinami L, Cunningham WE. Willingness to participate in HIV vaccine trials: The impact of trial attributes. Prevention Medicine. 2007; 44:554-557.

Newman PA, Duan N, Roberts KJ, Seiden D, Rudy ET, Swendeman D, Popova S. HIV vaccine trial participation among ethnic minority communities. Journal of Acquired Immune Deficiency Syndromes. 2006b; 41:210-217. [PubMed: 16394854]

Newman PA, Duan N, Rudy E, Roberts KJ, Swendeman D. Post-trial HIV vaccine adoption: Concerns, motivators and intentions among persons at risk for HIV. Journal of Acquired Immune Deficiency Syndromes. 2004; 37:1393-1403. [PubMed: 15483469]

Newman PA, Logie C. HIV vaccine acceptability: A systematic review and meta-analysis. AIDS. 2010; 24:1749-1756. [PubMed: 20597165]

Nichol KL, Mac Donald R, Hauge M. Factors associated with influenza and pneumococcal vaccination behavior among high-risk adults. Journal of General Internal Medicine. 1996; 11:673-677. [PubMed: 9120653]

Ottenberg AL, Wu JT, Poland GA, Jacobson RM, Koenig BA, Tilburt JC. Vaccinating health care workers against influenza: The ethical and legal rationale for mandate. American Journal of Public Health. 2011; 101:212-216. [PubMed: 21228284]

Rebmann T, Wright KS, Anthony J, Knaup RC, Peters EB. Seasonal influenza vaccine compliance among hospital-based and nonhospital-based healthcare workers. Infection Control and Hospital Epidemiology. 2012; 33:243-249. [PubMed: 22314061]

Rerks-Ngarm S, Pitisuttithum P, Nitayaphan S, Kaewkungwal J, Chiu J, Paris R, et al. Vaccination with ALVAC and AIDSVAX to prevent HIV-1 infection in Thailand. New England Journal of Medicine. 2009; 361:2209-2220. [PubMed: 19843557]

Rhodes T, Singer M, Bourgois P, Friedman SR, Strathdee SA. The social structural production of HIV risk among injecting drug users. Social Science \& Medince. 2005; 61:1026-1044.

Roberts KJ, Newman PA, Duan N, Rudy ET. HIV vaccine knowledge and beliefs among communities at elevated risk: Conspiracies, questions and confusion. Journal of the National Medical Association. 2005; 97:1662-1671. [PubMed: 16396058]

Rosenstock, IM.; Strecher, VJ.; Becker, MH. The health belief model and HIV risk behavior change.. In: DiClemente, RJ.; Peterson, JL., editors. Preventing AIDS: Theories and Methods of Behavioral Interventions. Plenum Press; New York: 1994. p. 5-22.

Salmon DA, Moulton LH, Omar SB, DeHart MP, Stokley S, Halsey NA. Factors associated with refusal of childhood vaccines among parents of school-aged children: A case-control study. Archives of Pediatrics \& Adolescent Medicine. 2005; 159:470-476. [PubMed: 15867122]

Shapiro CN. Epidemiology of hepatitis B. Pediatric Infectious Disease Journal. 1993; 12:433-437. [PubMed: 8392167]

Thomas PE, Voetsch AC, Song B, Calloway D, Goode C, Mundey L, et al. HIV risk behaviors and testing history in historically black college and university settings. Public Health Reports. 2008; 123:115-125. [PubMed: 19166095]

Workowski KA, Berman S. Sexually transmitted diseases treatment guidelines, 2010. Recommendations and Reports. 2010; 59:1-110. Retrieved from http://www.cdc.gov/mmwr/ preview/mmwrhtml/rr5912a1.htm?s_cid=rr5912a1_e. [PubMed: 21160459] 
Table 1

Associations of sociodemographic characteristics, vaccine attitudes, and compulsory HIV vaccine policy endorsement among a representative sample of persons attending likely dissemination venues in Los Angeles county ( $\mathrm{n}=1225)$

\begin{tabular}{|c|c|c|c|c|}
\hline \multirow[t]{2}{*}{ Characteristics } & \multirow[t]{2}{*}{ Total Sample (n=1225) } & \multicolumn{2}{|c|}{$\underline{\text { Endorse compulsory HIV vaccine policy }}{ }^{*}$} & \multirow[t]{2}{*}{ p-value } \\
\hline & & Yes $(n=590)$ & No $(n=635)$ & \\
\hline Age in years, mean (SE) & $36.8(0.55)$ & $36.4(0.64)$ & $37.2(0.75)$ & 0.400 \\
\hline \multicolumn{5}{|l|}{ Gender } \\
\hline Male & $55.6 \%$ & $43.3 \%$ & $56.7 \%$ & \\
\hline Female & $44.4 \%$ & $54.0 \%$ & $46.0 \%$ & 0.001 \\
\hline \multicolumn{5}{|l|}{ Race/ethnicity } \\
\hline Hispanic (English speaking) & $12.4 \%$ & $38.7 \%$ & $61.3 \%$ & \\
\hline Hispanic (Non-English speaking) & $37.6 \%$ & $60.5 \%$ & $39.5 \%$ & \\
\hline White & $17.7 \%$ & $30.3 \%$ & $69.7 \%$ & \\
\hline Black & $20.0 \%$ & $46.5 \%$ & $53.5 \%$ & \\
\hline Other & $12.3 \%$ & $47.5 \%$ & $52.5 \%$ & $<0.001$ \\
\hline \multicolumn{5}{|l|}{ Sexual orientation } \\
\hline Heterosexual & $78.8 \%$ & $50.8 \%$ & $49.2 \%$ & \\
\hline Gay/lesbian/bisexual & $21.2 \%$ & $38.0 \%$ & $62.0 \%$ & 0.002 \\
\hline \multicolumn{5}{|l|}{ Education } \\
\hline Less than high school & $29.5 \%$ & $61.8 \%$ & $38.2 \%$ & \\
\hline High school & $27.3 \%$ & $45.6 \%$ & $54.4 \%$ & \\
\hline Some college & $31.0 \%$ & $43.8 \%$ & $56.2 \%$ & \\
\hline Bachelors degree or above & $12.2 \%$ & $31.3 \%$ & $68.7 \%$ & $<0.001$ \\
\hline \multicolumn{5}{|l|}{ Have any children } \\
\hline Yes & $54.1 \%$ & $53.2 \%$ & $46.8 \%$ & \\
\hline No & $45.9 \%$ & $42.0 \%$ & $58.0 \%$ & 0.001 \\
\hline \multicolumn{5}{|l|}{ Monthly income } \\
\hline$\$ 0-\$ 500$ & $27.4 \%$ & $45.6 \%$ & $54.4 \%$ & \\
\hline$\$ 501-\$ 1000$ & $21.8 \%$ & $55.2 \%$ & $44.8 \%$ & \\
\hline$\$ 1001-\$ 2000$ & $27.1 \%$ & $51.8 \%$ & $48.2 \%$ & \\
\hline$>\$ 2000$ & $23.6 \%$ & $40.0 \%$ & $60.0 \%$ & 0.028 \\
\hline \multicolumn{5}{|l|}{ Health insurance } \\
\hline Private insurance & $20.8 \%$ & $41.9 \%$ & $58.1 \%$ & \\
\hline Public Insurance & $30.4 \%$ & $44.3 \%$ & $55.7 \%$ & \\
\hline No insurance & $48.8 \%$ & $53.0 \%$ & $47.0 \%$ & 0.022 \\
\hline Positive vaccine attitude scale (SE) & $3.01(0.01)$ & $3.09(0.02)$ & $2.94(0.02)$ & $<0.001$ \\
\hline Perceived HIV risk (SE) & $3.62(0.04)$ & $3.79(0.06)$ & $3.46(0.05)$ & $<0.001$ \\
\hline \multicolumn{5}{|c|}{ Have a regular place to go for medical care } \\
\hline Yes & $68.6 \%$ & $49.6 \%$ & $50.4 \%$ & \\
\hline No & $31.4 \%$ & $44.8 \%$ & $55.2 \%$ & 0.199 \\
\hline
\end{tabular}




\begin{tabular}{ccccc}
\hline Characteristics & Total Sample (n=1225) & \multicolumn{2}{c}{ Endorse compulsory HIV vaccine policy } & p-value \\
\cline { 3 - 4 } & & Yes (n=590) & No (n=635) & \\
\hline Yes & $25.7 \%$ & $34.1 \%$ & $65.9 \%$ & \\
No & $74.3 \%$ & $52.9 \%$ & $47.1 \%$ & $<0.001$ \\
\hline
\end{tabular}

Chi-square tests were conducted to compare the group differences in endorsement of compulsory HIV vaccine policy.

$S E$ Standard error; Means and percentages adjusted for primary sampling units, strata, and sampling weights.

* Compulsory HIV vaccine policy was a dichotomous variable derived from three Likert-scale variables. 


\section{Table 2}

Multivariable logistic regression examining predictors of compulsory HIV vaccination policy endorsement among a representative sample of persons attending likely dissemination venues in Los Angeles county $(\mathrm{n}=1225)$

\begin{tabular}{|c|c|c|c|}
\hline \multirow{2}{*}{$\begin{array}{l}\text { Variables } \\
\text { Age }\end{array}$} & \multirow{2}{*}{$\frac{\text { Odds Ratio (OR) }}{0.99}$} & \multicolumn{2}{|c|}{ 95\% Confidence Limits } \\
\hline & & 0.98 & 1.01 \\
\hline \multicolumn{4}{|l|}{ Gender } \\
\hline Female & 1.00 & -- & -- \\
\hline Male & 0.92 & 0.69 & 1.22 \\
\hline \multicolumn{4}{|l|}{ Race/ethnicity } \\
\hline White & 1.00 & -- & -- \\
\hline Hispanic (English speaking) & 1.06 & 0.64 & 1.76 \\
\hline Hispanic (Non-English speaking) ${ }^{*}$ & 1.69 & 1.03 & 2.76 \\
\hline Black & 1.53 & 0.91 & 2.56 \\
\hline Other ${ }^{*}$ & 1.74 & 1.01 & 3.03 \\
\hline \multicolumn{4}{|l|}{ Sexual orientation } \\
\hline Heterosexuals & 1.00 & -- & -- \\
\hline Gay/lesbian/bisexual & 0.84 & 0.56 & 1.26 \\
\hline \multicolumn{4}{|l|}{ Education } \\
\hline Bachelor's degree or above & 1.00 & -- & -- \\
\hline Less than high school ${ }^{* *}$ & 2.66 & 1.56 & 4.55 \\
\hline High school & 1.65 & 0.96 & 2.83 \\
\hline Some college ${ }^{*}$ & 1.71 & 1.03 & 2.86 \\
\hline Have any children & 1.13 & 0.79 & 1.63 \\
\hline \multicolumn{4}{|l|}{ Monthly income } \\
\hline$>\$ 2000$ & 1.00 & -- & -- \\
\hline$\$ 0-\$ 500$ & 1.22 & 0.74 & 2.00 \\
\hline$\$ 501-\$ 1000$ & 1.59 & 0.96 & 2.64 \\
\hline$\$ 1001-\$ 2000$ & 1.23 & 0.79 & 1.91 \\
\hline \multicolumn{4}{|l|}{ Health insurance } \\
\hline Private insurance & 1.00 & -- & -- \\
\hline Public insurance & 0.79 & 0.50 & 1.23 \\
\hline No insurance & 1.22 & 0.75 & 1.98 \\
\hline Positive vaccine attitude scale ${ }^{* *}$ & 2.48 & 1.70 & 3.61 \\
\hline Perceived HIV risk ${ }^{*}$ & 1.17 & 1.04 & 1.32 \\
\hline Have a regular place to go for medical care & 1.27 & 0.88 & 1.84 \\
\hline Injected drugs in the past 30 days ${ }^{*}$ & 0.66 & 0.45 & 0.97 \\
\hline
\end{tabular}

